\title{
Metastatic B-cell lymphoma masquerading as infectious retinitis and vasculitis
}

\author{
EMIL ANTHONY T. SAY, CHARLES L. KNUPP, KEVIN R. GERTSCH and SAI H. CHAVALA
}

\begin{abstract}
Kittner Eye Center, University of North Carolina Hospitals (EATS, KRG, SHC), Ambulatory Care Center, Chapel Hill, NC 27599-7040; Leo W. Jenkins Cancer Center, Eastern Carolina University (CLK), Greenville, NC 27834, USA
\end{abstract}

Received January 4, 2012; Accepted March 23, 2012

DOI: $10.3892 / 01.2012 .659$

\begin{abstract}
Intraocular lymphoma is a rare ocular malignancy that may occur in the retina or the uvea. Retina or vitreoretinal lymphoma accounts for the majority of cases and is often secondary to diffuse large B-cell lymphoma. In the present study, a 66-year-old Caucasian male with a history of Waldenstrom's macroglobulinemia with diffuse large B-cell lymphoma, presented with blurred vision in the left eye one month following cycle 4 of an R-CHOP regimen. At the time of onset, the patient was being treated for bacterial pneumonia. Visual acuity was 20/25 in his right eye (OD) and 20/30 in the left (OS). Ophthalmologic examination showed intraretinal white infiltrates associated with hemorrhage in the superotemporal midperiphery of the retina and vitritis OS. Initial diagnostic considerations included infectious (cytomegalovirus retinitis, syphilis, toxoplasmosis, tuberculosis), inflammatory (retinal vasculitis associated with autoimmune disease or hypercoagulable states) or malignant (intraocular lymphoma) diseases. The patient did not respond to intravitreal injection of foscarnet and oral valgancyclovir. Systemic work-up and aqueous fluid biopsy were inconclusive. Diagnostic vitrectomy yielded inconclusive results and the patient continued to have progressive loss of vision. A repeat diagnostic vitrectomy with retinal and subretinal biopsy confirmed large B cells consistent with metastatic B-cell lymphoma. A concomitant PET/CT scan was performed that revealed bilateral new pulmonary nodules resulting in additional chemotherapy. Our case shows the diagnostic dilemmas in patients with systemic lymphoma and the possible role of concurrent systemic restaging in patients with ocular complaints, even when in systemic remission.
\end{abstract}

Correspondence to: Dr Sai H. Chavala, Vitreo-Retina Service, 5151 Bioinformatics Bldg, CB no. 7040, Chapel Hill, NC 275997040, USA

E-mail: schavala@med.unc.edu

Key words: B-cell lymphoma, metastasis, uveitis, vasculitis, vitreoretinal lymphoma, vitrectomy

\section{Introduction}

Intraocular lymphoma is a rare ocular malignancy that may arise in the retina or the uvea. The exact incidence of intraocular lymphoma has not been reported, but it was previously estimated by Chan et al that at least 100 cases were diagnosed in a 3-year span during the past decade (1). Retina or vitreoretinal lymphoma accounts for the majority of cases and are often secondary to diffuse large B-cell lymphoma. These are highly aggressive tumors often with coexistent central nervous system (CNS) lymphoma (80\%), but rarely with systemic lymphoma (2). The tumors are bilateral in up to $90 \%$ of cases, and present as multiple creamy white or yellow intraretinal, subretinal or sub-RPE deposits with vitritis that can easily be mistaken for infectious or non-infectious uveitis. We describe herein a case of metastatic diffuse large B-cell lymphoma in a patient in apparent systemic remission. Institutional review board approval was not required for this case report. Informed consent was obtained from the patient for all diagnostic tests, surgery and treatment.

\section{Case report}

A 66-year-old Caucasian male presented with a one-month history of blurred vision in the left eye (OS). His past medical history was significant for hypertension and Waldenstrom's macroglobulinemia, which was diagnosed 5 years previously. The patient, who had a rash consistent with urticaria pigmentosa and an elevated serum tryptase level to perform bone marrow aspiration and biopsy to evaluate for systemic mastocytosis. No peripheral adenopathy or splenomegaly was present on examination. The marrow was inaspirable and the biopsy revealed extensive replacement by small lymphoplasmacytic cells and scattered mast cells consistent with reactive mast cells. Immunoglobulin stains for light and heavy chains were inconclusive, CD20 staining was positive and a diagnosis of Waldenstrom's macroglobulinemia was made. Serum protein electrophoresis demonstrated a monoclonal IgM paraprotein present at $3570 \mathrm{mg} / \mathrm{dl}$ (normal 40 to 230), $\kappa$ restricted. The patient was initially treated with rituximab but demonstrated no response in the IgM level after 6 months. He received a second course of rituximab, but again there was no response to the treatment. The patient was then started on rituximab, fludarabine and cyclophosphamide but was unable 
to complete the full course of therapy due to the development of marked pancytopenia requiring red blood cell and platelet transfusions. Bone marrow biopsy at that time revealed severe hypocellularity with residual involvement of Waldenstrom's macroglobulinemia. The IgM level had declined to $1330 \mathrm{mg} / \mathrm{dl}$ and remained stable as blood counts slowly improved over the following 3 years.

On a routine three-month follow-up visit, the patient was found to have a large left axillary mass, which he noted had developed three weeks earlier. He had no other symptoms. Fine needle aspiration was performed at that time and demonstrated large discohesive lymphocytes with prominent nucleoli. Flow cytometry demonstrated that the large lymphocytes expressed CD10, CD19 and CD20 with monoclonal $\kappa$ light chain restriction. A diagnosis of diffuse large B-cell lymphoma was made based on the microscopic and flow cytometric information. The serum IgM level was $1098 \mathrm{mg} / \mathrm{dl}$. The PET/CT fusion scan revealed bulky hypermetabolic bilateral axillary, retroperitoneal, right common iliac and inguinal adenopathy. Hypermetabolic activity was also present in the left cervical, supraclavicular, subpectoral, azygoesophageal recess and distal left external iliac node regions. Uptake was noted in a subpleural pulmonary nodule in the posterior basilar segment of the right lower lobe, which was suspicious for lymphoma. No other extranodal uptake was present. Serum lactate dehydrogenase level was elevated at 349 U/1 (normal, 118 to 273). Bilateral bone marrow biopsies revealed marrow involvement by a B-cell lymphoproliferative process, morphologically consistent with lymphoplasmacytic lymphoma (Waldenstrom's macroglobulinemia), which expressed monoclonal $\kappa$ light chain restriction but was CD 10 negative. No involvement by large B-cell lymphoma was observed. Lumbar puncture was not performed. Based on this information, the patient was considered to have at least stage IIIA disease but possibly stage IVA disease, due to the uptake in the subpleural nodule.

The patient was treated with R-CHOP and Pegfilgrastim but was hospitalized with neutropenia and a left upper lobe pulmonary infiltrate after cycle 2 . The CT scan revealed marked improvement in the adenopathy. Fever resolved on administration of antibiotics, but the patient had recurrent episodes of neutropenic fever despite chemotherapy dose reductions for cycles 3 and 4 with persistence of the left upper lobe pulmonary infiltrate. Moreover, Pseudomonas bacteremia was noted after cycle 4. Chemotherapy was postponed as the patient continued to experience fevers and malaise, was found to have significant narrowing of the superior segment of the lingula, and was treated with a prolonged course of piperacillin-tazobactam. A CT scan of the chest 3 months after the last chemotherapy revealed improvement of the left upper lobe pulmonary infiltrate and no pathological adenopathy. The serum IgM level was $548 \mathrm{mg} / \mathrm{dl}$. The pulmonary infiltrate had resolved by repeat $\mathrm{CT}$ scan of the chest 2 months later, with no pathological adenopathy present. Bone marrow aspiration and biopsy at that time revealed no involvement of large B-cell lymphoma but minimal persistent involvement of Waldenstrom's macroglobulinemia. Approximately one month after the patient received cycle 4 of his chemotherapy, and while under treatment for pneumonia, he noted a persistent painless blurring of vision in his left eye. The blurring did not improve, and was subsequently evaluated.
Visual acuity on initial presentation was 20/25 in the right eye (OD) and 20/30 OS. Intraocular pressures and anterior segment examination were normal in both eyes (OU) with the exception of the presence of 2+ khaki-colored cells in the anterior vitreous OS. Fundus examination OD was normal, whereas OS showed intraretinal infiltrates with associated hemorrhage in the superotemporal midperiphery and diffuse vitreous hemorrhage. Considering the immunocompromised state of the patient with regards to non-Hodgkin's lymphoma and chemotherapy, differential diagnoses included infectious [cytomegalovirus retinitis (CMV), syphilis, toxoplasmosis, tuberculosis], inflammatory (retinal vasculitis associated with autoimmune conditions) and malignant (metastatic systemic lymphoma) diseases.

Concern for possible CMV retinitis resulted in the initiation of oral valgancyclovir $900 \mathrm{mg}$ twice daily and one intravitreal injection of foscarnet $(2.4 \mathrm{mg} / 0.1 \mathrm{ml}) \mathrm{OS}$ prior to the results of diagnostic testing. Anterior chamber paracentesis and vitreous biopsy were performed and tested for toxoplasmosis, herpes simplex virus (HSV), varicella zoster virus (VZV), CMV, bacterial and fungal cultures, all of which were negative. Systemic work-up for tuberculosis (PPD, CXR), syphilis (RPR, FTA-ABS), Lyme disease and retinal vasculitis (ANCA, ANCA, ACE, PR3/MPO) were unremarkable, with the exception of a positive c-ANCA. A diagnostic pars plana vitrectomy was also performed to exclude metastatic lymphoma. Intraoperatively, diffuse vascular sheathing was observed, along with a chorioretinal scar inferior to the optic disc adjacent to an area of retinal whitening in addition to the retinal infiltrates with intraretinal hemorrhage in the superotemporal quadrant (Fig. 1A). Valgancyclovir was discontinued and the patient was referred to rheumatology for work up for possible ANCA-positive autoimmune disease associated with retinal vasculitis. Further testing with ANA, ENA, dsDNA, APLA, C3/C4, ESR, CRP, CBC, creatinine and brain MRI/MRA were negative, with the exception of a mildly elevated ESR and a low WBC count.

There was additional concern for possible reactivated ocular toxoplasmosis associated with the chorioretinal scar observed intraoperatively, and the patient was also started on oral trimethoprim-sulfamethoxazole $(800 \mathrm{mg} / 160 \mathrm{mg})$ twice daily. Two weeks later, vision remained stable at 20/30, but new yellow subretinal infiltrates appeared adjacent to the scar. The patient was administered an injection of intravitreal clindamycin $(1 \mathrm{mg} / 0.1 \mathrm{ml})$ and dexamethasone $(400 \mu \mathrm{g} / 0.1 \mathrm{ml})$ for suspected worsening of ocular toxoplasmosis. One week following the intravitreal injection, the previously noted chorioretinal scar was not a scar but an elevated lesion with presence of subretinal fluid, and new infiltrates were observed from the nasal to the optic disc and confirmed by optical coherence tomography (Fig. 1B). Concern regarding an indolent fungal infection resulted in the administration of oral Levaquin and voriconazole, but without improvement. The visual acuity of the patient decreased to 20/250, and the retinal infiltrates continued to increase in size following one week of treatment (Fig. 1C). A second pars plana vitrectomy was applied with subretinal biopsy 3 months after the initial presentation. Cytopathology and flow cytometry confirmed large B-cell lymphoma, consistent with pathology observed on a previous axillary node biopsy, leading to a diagnosis of metastatic B-cell 


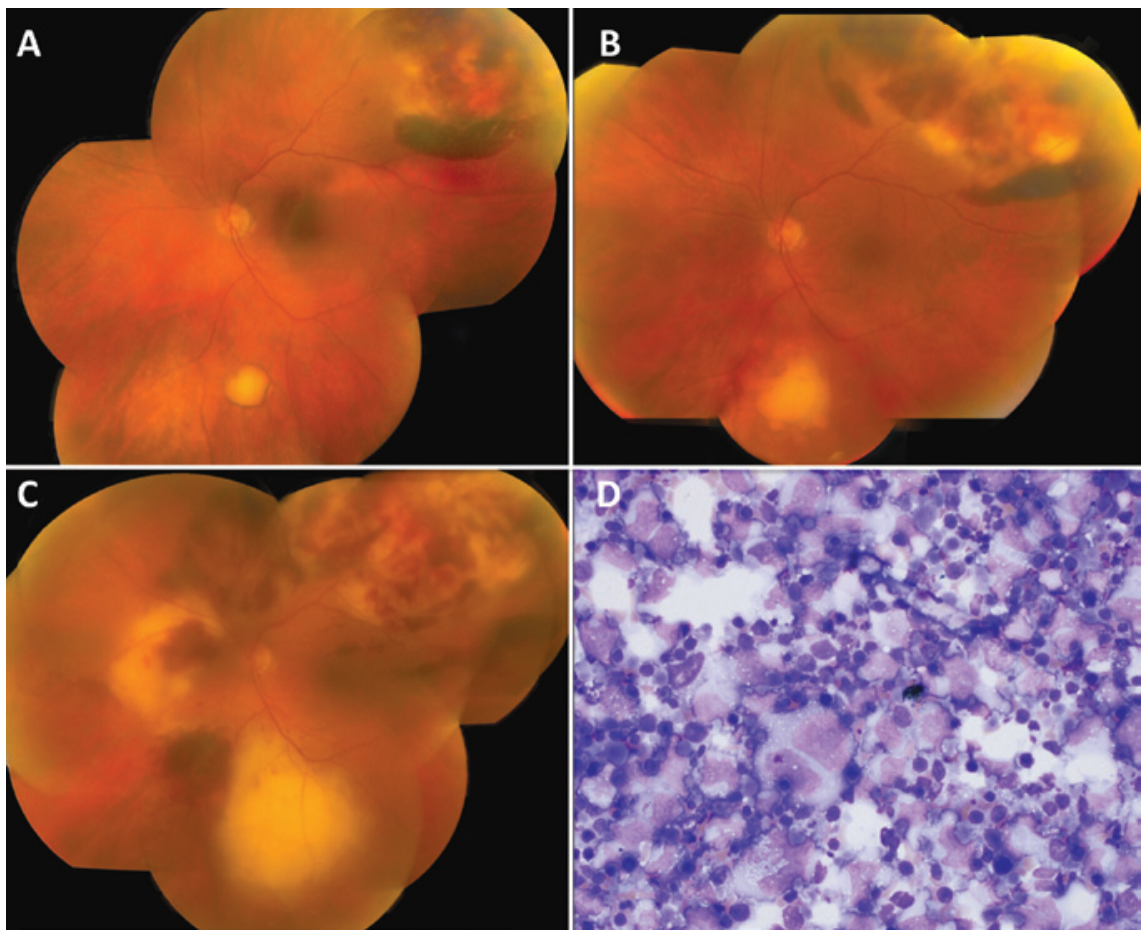

Figure 1. A 66-year-old male presented with a one-month history of blurred vision in his left eye (OS). (A) Following a diagnostic vitrectomy to remove diffuse vitreous hemorrhage, a fundus examination revealed white intraretinal infiltrates associated with intraretinal hemorrhages in the superotemporal periphery and a new white choroidal mass with pigmented borders inferior to the optic nerve. (B) Three weeks and (C) 4 weeks following the first diagnostic vitrectomy, there was a progressive and rapid increase in the size of the infiltrates in the superotemporal periphery as well as growth of the choroidal mass. (C) New chorioretinal infiltrates were observed 4 weeks following vitrectomy, resulting in a second diagnostic vitrectomy with subretinal biopsy. (D) Cytopathology and flow cytometry of vitreous and subretinal biopsy confirmed large B-cell lymphoma comparable to pathology observed on a previous axillary node biopsy.

lymphoma (Fig. 1D). A restaging work-up for lymphoma with $\mathrm{PET} / \mathrm{CT}$ fusion scan revealed a hypermetabolic left lung nodule measuring $1.9 \times 1.7 \mathrm{~cm}$, consistent with active lymphoma. No other involvement in lymph nodes or other extranodal sites, including the central nervous system was observed. Lumbar puncture was not performed at this time.

The patient then underwent external beam radiotherapy with a total dose of 3000 cGy to the left eye in 200 cGy fractions. Final visual acuity was no light perception OS. At the conclusion of the radiation therapy, repeat PET/CT scan, as compared to the scan at the original diagnosis of large B-cell lymphoma a year earlier, demonstrated resolution of the activity in the previously involved nodal sites and the small right subpleural pulmonary nodule, but new extensive bilateral hypermetabolic pulmonary nodules, the largest measuring $5 \mathrm{~cm}$ with extensive hypermetabolic mediastinal and bilateral hilar adenopathy. No extrathoracic hypermetabolic activity was present. The patient was evaluated as a candidate for autologous stem cell transplantation but declined due to difficulty tolerating the previous R-CHOP chemotherapy; however, the patient agreed to rituximab and a reduced dose of bendamustine therapy at $70 \mathrm{mg} / \mathrm{m}^{2}$. The patient tolerated the first cycle well with an expected decrease in blood counts, and the dose of bendamustine was increased to $100 \mathrm{mg} / \mathrm{m}^{2}$ for cycle 2 of therapy. He developed significant diarrhea after this treatment but did not require hospitalization. PET/CT scan after cycle 2 of treatment demonstrated interval resolution of hypermetabolic mediastinal adenopathy and marked improvement in the activity in the lungs with only low-level activity remaining at the site of the largest pulmonary nodule, which had decreased from $5 \mathrm{~cm}$ to a $1.5 \mathrm{~cm}$ wedge-shaped density. The patient has continued on the rituximab and bendamustine chemotherapy, but the bendamustine dose was reduced to $70 \mathrm{mg} / \mathrm{m}^{2}$ without significant toxicities. The patient is presently alive after 6 cycles of this therapy with Eastern Cooperative Oncology Group (ECOG) performance status 1.

\section{Discussion}

Intraocular lymphoma is often associated with a grim prognosis due to associated central nervous system disease. In a review by Mochizuki and Singh, ocular-CNS lymphoma was found to occur in $61 \%$ of patients, primary intraocular lymphoma in $17 \%$, ocular-visceral lymphoma in $17 \%$ and ocular-visceralCNS lymphoma in $5 \%$ of patients (2). Intraocular lymphoma from the systemic dissemination of visceral lymphoma is usually confined to the choroid and infrequently presents as vitreoretinal lymphoma, similar to our case (3). The rarity of intraocular lymphoma, its diverse clinical presentation, the ability to masquerade as different uveitic entities, and in this case, an atypical site of ocular involvement, makes diagnosis difficult. Additionally, successful confirmatory biopsy by fine needle aspiration or vitrectomy is a demanding procedure that requires significant skill not only by the ophthalmologist, but also the cytopathologist. These factors often cause a delay in the diagnosis and initiation of treatment. A report by Karma et al showed a median of 8 months after onset of symptoms to the first ocular examination, and a median of 13 months from onset of symptoms until diagnosis of intraocular lymphoma (4). 
Our patient presented with unilateral blurred vision from vitreous hemorrhage accompanied by progressive increase in size of the retinal infiltrates. Given the patient's history of neoplastic disease and his immunocompromised state while on chemotherapy, important differential diagnoses included infectious and non-infectious entities. The retinal infiltrates and vitreous hemorrhage were both atypical for intraocular lymphoma secondary to systemic metastases, as metastatic systemic lymphoma more commonly presents with uveal involvement alone. The positive c-ANCA associated with retinal infiltrates and hemorrhages was also evaluated for a systemic vasculitis with ocular involvement. ANCA positivity in our case was most likely secondary to systemic lymphoma, which has previously been reported (5). Although vitreous and aqueous biopsies for viral, bacterial and fungal infections, as well as cytopathology for lymphoma cells were negative, empiric antibiotic therapy for infectious retinitis was indicated considering the sight-threatening nature of the disease. Initially, CMV retinitis was considered the most likely cause due to the presence of retinal infiltrates with adjacent subretinal, intraretinal and vitreous hemorrhage, which is not commonly associated with ocular lymphoma, particularly when the patient was systemically in remission from lymphoma. Following a negative response to treatment, a diagnostic vitrectomy was performed, revealing a new chorioretinal scar inferior to the optic disc. A diagnosis of toxoplasmosis appeared likely, and appropriate systemic and intravitreal antibiotic-steroid regimens were administered, again without response and with progression. It was not until subretinal biopsy with pars plana vitrectomy was performed that a diagnosis of metastatic lymphoma was confirmed, triggering a search for systemic metastases, which was positive. This was 3 months after the patient's first ophthalmology evaluation and 4 months after the 4 th cycle of R-CHOP.
With hindsight, a concurrent systemic staging along with an earlier subretinal biopsy may have led to an earlier diagnosis and a better visual outcome. The relapsing clinical course of our patient may have led us to perform more aggressive diagnostic procedures early on, in addition to coordinating a repeat PET/ CT scan and lumbar puncture with his oncologist.

Our case showed the difficulty in obtaining confirmatory diagnosis in metastatic intraocular lymphoma, and possibly a role for concurrent systemic restaging, even in patients with ocular complaints and in systemic remission. More importantly, a strong clinical suspicion should always be present and the importance of repeated diagnostic biopsies should be emphasized, particularly in an aggressive malignancy with a significant threat to life.

\section{Acknowledgements}

Support was provided by an unrestricted grant from Research to Prevent Blindness.

\section{References}

1. Chan CC, Buggage RR and Nussenblatt RB: Intraocular lymphoma. Curr Opin Ophthalmol 13: 411-418, 2002.

2. Mochizuki M and Singh AD: Epidemiology and clinical features of intraocular lymphoma. Ocul Immunol Inflamm 17: 69-72, 2009.

3. Coupland SE and Damato B: Understanding intraocular lymphoma. Clin Experiment Ophthalmol 36: 564-578, 2008.

4. Karma A, von Willebrand EO, Tommila PV, Paetau AE, Oskala PS and Immonen IJ: Primary intraocular lymphoma: improving the diagnostic procedure. Ophthalmology 114: 1372-1377, 2007.

5. Lee AS, Wiesner O, Gillespie DJ, Witzig TE, Homburger $\mathrm{H}$ and Specks U: A 70-year-old man with pulmonary infiltrates and a positive antineutrophil cytoplasmic autoantibody test result. Chest 127: 1045-1050, 2005. 